\title{
Entwicklung einer Bewertungsskala für den Sino-Nasal Outcome Test-20 German Adapted Version (SNOT-20 GAV)
}

\author{
Ingo Baumann ${ }^{1}$ und Harry De Maddalena ${ }^{2}$

\section{Universitäts-HNO-Klinik Heidelberg \\ 2 Universitäts-HNO-Klinik Tübingen}

\section{Hintergrund}

- die chronische Rhinosinusitis (CRS) betrifft ca. 5\% der Bevölkerung.

- der Sino-Nasal Outcome Test-20 German Adapted Version (SNOT-20 GAV) ist ein validiertes Messinstrument zur Messung der gesundheitsbezogenen Lebensqualität bei Patienten mit CRS.

- bisher existieren keine Normwerte und kein klinischer Bewertungsmaßstab

Patienten und Methoden

\section{Vergleichskollektiv:}

webbasierte Online-Befragung der Mitarbeiter des Universitätsklinikums Heidelberg

ca. 7000 Emails,

778 Probanden, 510 Frauen, 268 Männer, Ratio 1,9:1

Alter 38,4 Jahre; Spannweite 17 - 67 Jahre

Operiertes Kollektiv

163 Patienten, 55 Frauen, 108 Männer, Ratio 0,51:1) Alter 46,4 Jahre; Spannweite 17,6 - 80,4 Jahre

Erstoperation bei 125 (78\%) Patienten

zusätzliche Septumplastik bei 120 (74\%) Patienten

beidseitige Ethmoidektomie bei 113 (70\%) Patienten,

beidseitige Pansinusoperation bei 44 (27\%) Patienten

Kollektivbildung bei Probanden und Patienten

W-CRS ( $n=601)$ : $\quad$ Probanden ohne CRS

(anamnestisch, Vergleichskollektiv)

P-CRS ( $n=177)$ : $\quad$ Probanden mit möglicherweise oder sicher bestehender CRS

(anamnestisch, Vergleichskollektiv)

E-CRS ( $n=163)$ : bestehende CRS bei Patienten (operiertes Kollektiv)

Lebensqualitätsmessung

Messung der gesundheitsbezogenen Lebensqualität mit dem SNOT-20 GAV

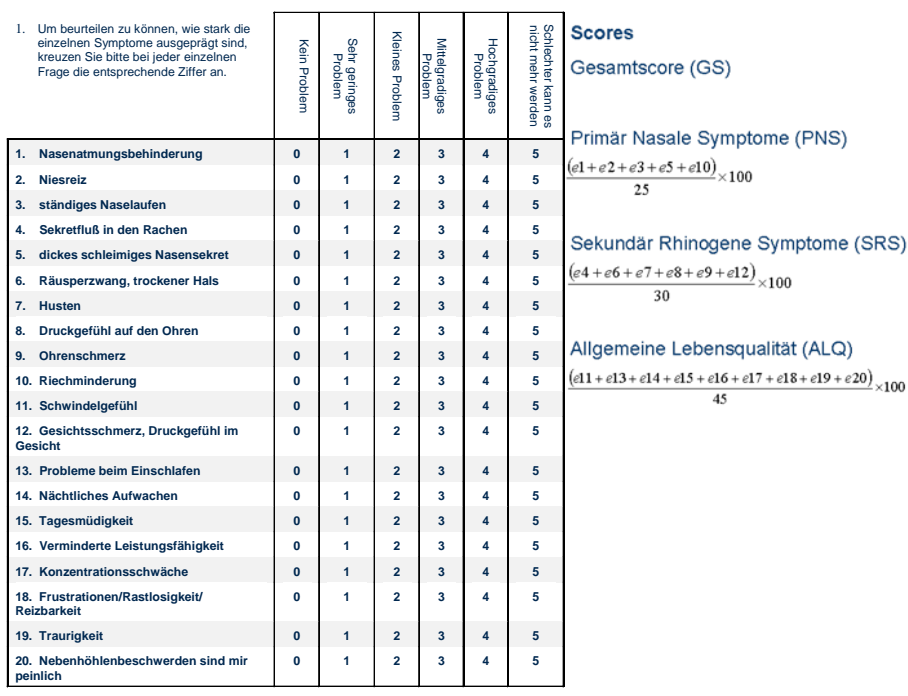

Ergebnisse

Konstruktion der Bewertungsskala

Skala PNS wurde als relevante Skala identifiziert: -weist annähernde Normalverteilung für E-CRS auf -Standardabweichungen von E-CRS treffen fast exakt die Mittelwerte von P-CRS und W-CRS (siehe Abb. 2) -Festlegung der Beschwerdegrade in Bezug zu diesen Werten (siehe Tab. 1)

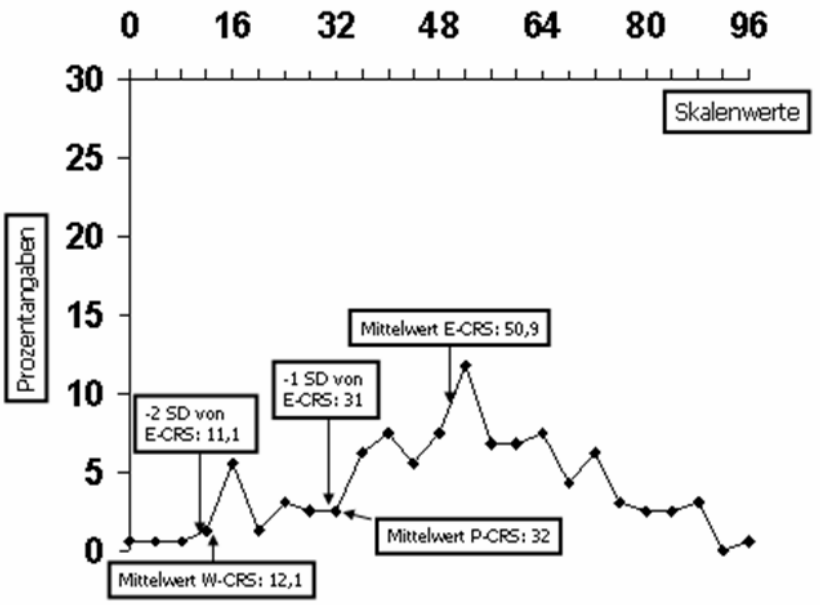

Abb. 2: Verteilung der Rohwerte, Mittelwerte und Standardabweichungen (SD) in der Skala „Primäre Nasale Symptome" bei operierten Patienten mit vorhandener CRS (ECRS) sowie Mittelwerte der Patientengruppen mit potentiell vorhandener CRS (P-CRS) und nicht vorhandener CRS (WCRS)

\begin{tabular}{|c|c|c|}
\hline $\begin{array}{c}\text { Beschwerde- } \\
\text { klasse }\end{array}$ & $\begin{array}{c}\text { Skalenwerte } \\
\text { PNS }\end{array}$ & Beschwerdegrad \\
\hline I & $<=12$ & $\begin{array}{c}\text { keine bis geringgradige } \\
\text { Beschwerden }\end{array}$ \\
\hline II & $>12$ und $<=32$ & $\begin{array}{c}\text { gering- bis mittelgradige } \\
\text { Beschwerden }\end{array}$ \\
\hline III & $>32$ und $<=52$ & $\begin{array}{c}\text { mittel- bis hochgradige } \\
\text { Beschwerden }\end{array}$ \\
\hline IV & $>52$ & $\begin{array}{c}\text { hochgradige und } \\
\text { höchstgradige Beschwerden }\end{array}$ \\
\hline
\end{tabular}

Tab. 1: Beschwerdeklassen mit Beschreibung des Beschwerdegrades für die Skala PNS Schlussfolgerungen

Mit der Bewertungsskala für die Skala Primär Nasale Symptome (PNS) des SNOT-20 GAV kann eine Bewertung der Beschwerden der Patienten in Bezug auf ein Normkollektiv vorgenommen werden. Damit wird die Einordnung der Beschwerdeausprägung für den Arzt und den Patienten erleichtert. Zukünftige Untersuchungen müssen zeigen, ob dieses Instrument bei der Indikationsstellung für die Therapie hilfreich sein kann. 